\title{
Lyapunov-Krasovskii Functionals for Predictor Feedback Control of Linear Systems with Multiple Input Delays
}

\author{
Zhao-Yan $\mathrm{Li}^{1}$ Bin Zhou ${ }^{2}$ James Lam ${ }^{3}$ \\ 1. Department of Mathematics, Harbin Institute of Technology, Harbin, 150001, P. R. China. \\ E-mail: zhaoyanlee@gmail.com; lizhaoyan@hit.edu.cn. \\ 2. Center for Control Theory and Guidance Technology, Harbin Institute of Technology, Harbin, 150001, China. \\ E-mail: binzhoulee@163.com, binzhou@hit.edu.cn \\ 3. Department of Mechanical Engineering, University of Hong Kong, Hong Kong. \\ E-mail: james.lam@hku.hk
}

\begin{abstract}
This paper is concerned with the Lyapunov-Krasovskii functional construction of linear control systems with multiple input delays. By transforming the predictor feedback control systems into a delay-free linear system with external inputs, a Lyapunov-Krasovskii functional is constructed in terms of a set of linear matrix inequalities (LMIs). It is shown that the solvability of this set of LMIs is equivalent to the asymptotic stability of the delay-free linear system induced from the predictor feedback control system. The proposed Lyapunov-Krasovskii functional is also found to be an ISS Lyapunov-Krasovskii functional for the predictor feedback control systems. An example is worked out to validate the effectiveness of the proposed method.
\end{abstract}

Key Words: Asymptotic stability; Lyapunov-Krasovskii functionals; Predictor feedback; Time-delay systems.

\section{Introduction}

Dynamic systems with time delays have received more and more attention because time-delay systems have many applications in practice engineering such as network control system, chemical process control, population model for instance. Since the stability and stabilization problems of time-delay systems are very important in both theory and practice, considerable research efforts were focused on them and many important related results have been obtained in the literature (see [7], [9], [11] and the references therein). Nevertheless there are still many unsolved problems in the stability analysis and stabilization of time-delay systems because of their infinite dimensional nature.

In the existing results regarding stability and stabilization of time-delay systems, most of them deal with linear systems with state delays (see, for example, [8], [16], and [17]) while relatively few results are available for control problems of linear systems with input delays. To deal with the stabilization of control systems with input delays, there are basically two efficient design methods, namely, memory controller$\mathrm{s}$ design by predictor feedback and memoryless controllers design adopted from delay-free systems. Memoryless controllers have been utilized by many researchers, for example, [3] and [5]. The advantage of this kind of methods is that they are very easy to implement. However, this kind of methods may fail if the delays are too large. In contrast, the predictor feedback that was originated by [13] can allow arbitrarily large input delays. The basic idea of this approach is to transform the delay system into an equivalent delayfree system for which any conventional design approaches are applicable. This method has been widely investigated

This work was partially supported by the National Natural Science Foundation of China under Grant numbers 61104124, 61273028 and 61322305 , by the Fundamental Research Funds for the Central Universities under Grant HIT.NSRIF.2011007, by Program for Innovation Research of Science in Harbin Institute of Technology (PIRS of HIT A201407), and by GRF HKU 7137/13E and SZSTI JCYJ20120831142942514 in the literature and has received renewed interest in recent years (see, for example, [1], [12], and [18]).

It is well known that in the stability analysis of time-delay systems, Lyapunov-Krasovskii functional is one of the most important tools. Many results about the stability and stabilization of the time-delay systems can be derived by using Lyapunov-Krasovskii functionals (see [10] and the references therein). To analyse the exponential stability of the linear predictor feedback system with a single time-varying input delay, a time-varying Lyapunov-Krasovskii functional was constructed based on the backstepping method for partial differential equations in [6]. By using a transformation of the actuator states, the Lyapunov-Krasovskii functional of the linear predictor feedback system with distributed input delays was obtained in [2]. The linear systems with pointwise and distributed input delays was considered in [14] and the ISS Lyapunov-Krasovskii functionals defined by [15] was constructed for linear systems with pointwise and distributed input delays. The proposed Lyapunov-Krasovskii functionals are helpful in the analysis of the ISS property of the closed-loop time-delay systems.

In this paper, we will study the exponential stability of linear systems with multiple input delays by constructing suitable Lyapunov-Krasovskii functionals. To this end, we first transform the closed-loop time-delay system with predictor feedback into an equivalent delay-free system. Then, based on the delay-free linear system, a Lyapunov-Krasovskii functional guaranteeing the exponential stability of the original time-delay system is proposed in terms of the solvability of a set of linear matrix inequalities (LMIs). It is proven that the set of LMIs provided is always solvable provided the delay-free linear system is asymptotically stable. At the same time, we show that the obtained Lyapunov-Krasovskii functional is also an ISS Lyapunov-Krasovskii functional for the predictor feedback control system. A numerical example is worked out to show the effectiveness of the proposed approach. The merit of the proposed approach is that the construction of the Lyapunov-Krasovskii functional does not 
need the backstepping method for partial differential equation as used in [6] and is simply based on some LMIs which can be efficiently solved by the existing software package.

Notation: For any given matrix $A \in \mathbf{R}^{n \times m}$, we respectively use $A^{\mathrm{T}}$ and $\lambda(A)$ to denote its transpose and eigenvalue set. For a positive definite matrix $P$, the symbol $\lambda_{\min }(P)$ and $\lambda_{\max }(P)$ denote respectively its minimal and maximal eigenvalues. For two positive integers $p$ and $q$, we use $\mathbf{I}[p, q]$ to present the set $\{p, p+1, \ldots q\}$. The symbol $|\cdot|$ refers to the Euclidean norm. Let $\tau>0$ is a given real number. $\mathscr{C}_{n, \tau}=\mathbf{C}\left([-\tau, 0], \mathbf{R}^{n}\right)$ present the Banach space of continuous functions mapping the interval $[-\tau, 0]$ into $\mathbf{R}^{n}$ with the topology of uniform convergence.

\section{Problem Formulation and Preliminary}

In this paper, we consider the following linear system with multiple input delays

$$
\dot{x}(t)=A x(t)+B_{0} u(t)+\sum_{i=1}^{p} B_{i} u\left(t-\tau_{i}\right), \quad \forall t \geq 0,
$$

where $A \in \mathbf{R}^{n \times n}, B_{i} \in \mathbf{R}^{n \times m}, i \in \mathbf{I}[0, p]$ are constant matrices and $\tau_{i}>0, i \in \mathbf{I}[1, p]$ are constants. Without loss of generality, we assume that $\tau_{1} \leq \tau_{2} \leq \cdots \leq \tau_{p} \triangleq \tau$. For stabilization of system (1), the predictor feedback can be designed as follows [19]:

$$
u(t)=F\left(x(t)+\sum_{i=1}^{p} \int_{t-\tau_{i}}^{t} \mathrm{e}^{A\left(t-\theta-\tau_{i}\right)} B_{i} u(\theta) \mathrm{d} \theta\right)
$$

which is such that the closed-loop system

$$
\left\{\begin{array}{l}
\dot{x}(t)=A x(t)+B_{0} u(t)+\sum_{i=1}^{p} B_{i} u\left(t-\tau_{i}\right), \\
u(t)=F\left(x(t)+\sum_{i=1}^{p} \int_{t-\tau_{i}}^{t} \mathrm{e}^{A\left(t-\theta-\tau_{i}\right)} B_{i} u(\theta) \mathrm{d} \theta\right)
\end{array}\right.
$$

has the characteristic equation $\Delta(s)=$ $\operatorname{det}\left(s I_{n}-A-B F\right)$ that has only a finite number of zeros. Here

$$
B=B_{0}+\mathscr{B}, \mathscr{B}=\sum_{i=1}^{p} \mathrm{e}^{-A \tau_{i}} B_{i} .
$$

Hence the stability of the closed-loop system (3) is guaranteed if $(A, B)$ is stabilizable and $F$ is well designed.

It is well known that Lyapunov-Krasovskii functionals play an important role in the analysis and design of control systems, especially, in the stability analysis of control systems. For the closed-loop system in (3), though the stability can be asserted by its characteristic equation, a LyapunovKrasovskii functional is not available. In this paper, we are interested in the construction of a Lyapunov-Krasovskii functional for this class of time-delay systems. The difficulty of the problem lies in the fact that the state of the closed-loop system should be considered as $\left(x(t), u_{t}\right) \in \mathbf{R}^{n} \times \mathscr{C}_{m, \tau}$, but not $x_{t} \in \mathscr{C}_{n, \tau}$ [6]. To make this clear, we give the following definition and lemma.

Definition 1 [6] The closed-loop system (3) is exponentially stable if there exist positive constants $G$ and $g$ such that

$$
|x(t)|^{2}+\left\|u_{t}\right\|^{2} \leq G \mathrm{e}^{-g t}\left(|x(0)|^{2}+\left\|u_{0}\right\|^{2}\right),
$$

where

$$
\left\|u_{t}\right\|^{2}=\int_{t-\tau}^{t}|u(\theta)|^{2} \mathrm{~d} \theta .
$$

Lemma 1 If there exist constants $\alpha_{i}>0, i=1,2,3$, such that the functional $V: \mathbf{R}^{+} \times \mathbf{R}^{n} \times \mathscr{C}_{m, \tau} \rightarrow \mathbf{R}^{+}$satisfies the following two conditions:

$$
\begin{aligned}
& \text { 1) } \alpha_{1}\left(|x(t)|^{2}+\left\|u_{t}\right\|^{2}\right) \leq V\left(t, x(t), u_{t}\right) \leq \\
& \alpha_{2}\left(|x(t)|^{2}+\left\|u_{t}\right\|^{2}\right) \\
& \text { 2) } \dot{V}\left(t, x(t), u_{t}\right) \leq-\alpha_{3}\left(|x(t)|^{2}+\left\|u_{t}\right\|^{2}\right) \text {; }
\end{aligned}
$$

then the state $\left(x(t), u_{t}\right)$ satisfies (5) for some constants $G$ and $g$. In this case, $V$ is called as a Lyapunov-Krasovskii functional for the closed-loop system (3).

Proof. Obviously, the following inequality

$$
-\alpha_{2}\left(|x(t)|^{2}+\left\|u_{t}\right\|^{2}\right) \leq-V\left(t, x(t), u_{t}\right),
$$

holds true by Item 1 . With this and Item 2 we have

$$
\begin{aligned}
\dot{V}\left(t, x(t), u_{t}\right) & \leq-\alpha_{3}\left(|x(t)|^{2}+\left\|u_{t}\right\|^{2}\right) \\
& \leq-\frac{\alpha_{3}}{\alpha_{2}} V\left(t, x(t), u_{t}\right) .
\end{aligned}
$$

Therefore, it is follows that

$$
\begin{aligned}
|x(t)|+\left\|u_{t}\right\| & \leq \frac{1}{\alpha_{1}} V\left(t, x(t), u_{t}\right) \\
& \leq \frac{1}{\alpha_{1}} \mathrm{e}^{-\frac{\alpha_{3}}{\alpha_{2}} t} V\left(0, x(0), u_{0}\right) \\
& \leq \frac{\alpha_{2}}{\alpha_{1}} \mathrm{e}^{-\frac{\alpha_{3}}{\alpha_{2}} t}\left(|x(0)|^{2}+\left\|u_{0}\right\|^{2}\right),
\end{aligned}
$$

i.e., the closed-loop system (3) is exponential stable in the sense of Definition 1.

So in this paper we will construct a Lyapunov-Krasovskii functional that satisfies the conditions in Lemma 1. To this end, we firstly introduce a new state variable as

$$
\begin{aligned}
& z(t)=x(t)+\omega(t), \\
& \omega(t)=\sum_{i=1}^{p} \int_{t-\tau_{i}}^{t} \mathrm{e}^{A\left(t-\theta-\tau_{i}\right)} B_{i} u(\theta) \mathrm{d} \theta,
\end{aligned}
$$

by which the original time-delay system (1) can be expressed as

$$
\dot{z}(t)=A z(t)+B u(t), \quad t \geq 0,
$$

which is a delay-free linear system. Hence the feedback (2) becomes

$$
u(t)=F z(t),
$$

and the closed loop system (3) reads

$$
\left\{\begin{array}{l}
\dot{z}(t)=A z(t)+B u(t) \\
u(t)=F z(t) \\
x(t)=z(t)-\omega(t)
\end{array}\right.
$$

So we only need to find the Lyapunov-Krasovskii functional of system (14).

At the end of this section, we introduce some technical lemmas that will be used in the next section. 
Lemma 2 For any constant matrix $M \in \mathbf{R}^{n \times n}, M=$ $M^{\top}>0$, integers $\gamma_{1}, \gamma_{2}$ with $\gamma_{1} \geq \gamma_{2}$, vector function $\omega: \mathbf{I}\left[\gamma_{1}, \gamma_{2}\right] \rightarrow \mathbf{R}^{n}$ such that the sums in the following are all well defined, then

$$
\begin{aligned}
& \left(\sum_{i=\gamma_{1}}^{\gamma_{2}} \omega(i)\right)^{\top} M\left(\sum_{i=\gamma_{1}}^{\gamma_{2}} \omega(i)\right) \\
& \leq\left(\gamma_{2}-\gamma_{1}+1\right) \sum_{i=\gamma_{1}}^{\gamma_{2}} \omega^{\top}(i) M \omega(i) .
\end{aligned}
$$

Lemma 3 [4] Given $h>0$, for a vector-valued function $a(\theta)$ and $Z>0$ of appropriate dimensions, there holds

$$
\begin{aligned}
& \left(\int_{-h}^{0} a(\theta) \mathrm{d} \theta\right)^{\top} Z\left(\int_{-h}^{0} a(\theta) \mathrm{d} \theta\right) \\
& \leq h \int_{-h}^{0} a^{\top}(\theta) Z a(\theta) \mathrm{d} \theta .
\end{aligned}
$$

Lemma 4 (Schur Complement Lemma) For any matrix $\Phi=$ $\Phi^{\mathrm{T}}$ of the form

$$
\Phi=\left[\begin{array}{cc}
A & B \\
B^{\top} & C
\end{array}\right]
$$

if $C$ is invertible, then $\Phi<0$ if and only if $C<0$ and $A-B C^{-1} B^{\top}<0$.

\section{Main Results}

Before giving our main results, according to the discussion in the above section, we impose the following assumption on the closed-loop system (3).

Assumption 1 The matrix $H=A+B F$ is Hurwitz, namely, all the eigenvalues of $H$ have negative real parts.

Then we have the following result regarding the construction of a Lyapunov-Krasovskii functional for the closed-loop system (3) or (14).

Theorem 1 Let Assumption 1 hold true and $\alpha>0$ be some constant such that

$$
\operatorname{Re}\left\{\lambda_{j}\left(A-\frac{\alpha}{2} I_{n}\right)\right\}<0, j \in \mathbf{I}[1, n]
$$

Then the following two statements hold true.

1) There exist a positive definite matrix $P=\left[P_{i j}\right] \in$ $\mathbf{R}^{2 n \times 2 n}$ with $P_{i j} \in \mathbf{R}^{n \times n}, i, j=1,2$, and a positive definite matrix $R \in \mathbf{R}^{n \times n}$ such that the following LMIs,

$$
\begin{aligned}
& \Delta=P_{12}^{\top} B_{0} F+F^{\boldsymbol{\top}} B_{0}^{\top} P_{12}+P_{22}(A+\mathscr{B} F) \\
& +\left(A^{\boldsymbol{\top}}+F^{\boldsymbol{\top}} \mathscr{B}^{\boldsymbol{\top}}\right) P_{22}+\mathscr{R}<0, \\
& \Gamma(i)=\left[\begin{array}{ccc}
\Gamma_{11}(i) & \Gamma_{12} & \Gamma_{13}(i) \\
\Gamma_{12}^{\top} & \Gamma_{22} & P_{12}^{\top}-P_{22} \\
\Gamma_{13}^{\top}(i) & P_{12}-P_{22} & \Gamma_{33}(i)
\end{array}\right]<0
\end{aligned}
$$

hold true, where $i \in \mathbf{I}[1, p]$ and

$$
\begin{aligned}
& \Gamma_{11}(i)=\frac{1}{p \tau_{i}}\left(P_{11}\left(A+B_{0} F\right)+\left(A+B_{0} F\right)^{\top} P_{11}\right. \\
& \left.+F^{\boldsymbol{\top}} \mathscr{B}^{\top} P_{12}^{\top}+P_{12} \mathscr{B} F+\mathscr{R}\right), \\
& \Gamma_{12}=A^{\top} P_{12}+P_{12} A+P_{11} B_{0} F+P_{12} \mathscr{B} F \\
& +F^{\boldsymbol{\top}} B_{0}^{\boldsymbol{\top}} P_{12}+F^{\top} \mathscr{B} \boldsymbol{\top} P_{22}+\mathscr{R}, \\
& \Gamma_{13}(i)=\frac{1}{p \tau_{i}}\left(P_{11}-P_{12}\right), \\
& \Gamma_{22}=\mathrm{e}^{-\alpha \tau}\left(\left(A-\frac{\alpha}{2} I_{n}\right)^{\top} R+R\left(A-\frac{\alpha}{2} I_{n}\right)\right), \\
& \Gamma_{33}(i)=-\frac{1}{p^{2} \tau_{i}} \mathrm{e}^{-\alpha \tau} R, \\
& \mathscr{R}=\sum_{i=1}^{p} F^{\boldsymbol{\top}} B_{i}^{\boldsymbol{\top}} \mathrm{e}^{-A^{\top} \tau_{i}} R \mathrm{e}^{-A \tau_{i}} B_{i} F .
\end{aligned}
$$

2) If $P>0$ and $R>0$ satisfy (19), (20) and (22), then the functional

$$
\begin{aligned}
& V\left(x(t), u_{t}\right)=\left[\begin{array}{c}
x(t) \\
\omega(t)
\end{array}\right]^{\top} P\left[\begin{array}{c}
x(t) \\
\omega(t)
\end{array}\right] \\
& +\sum_{i=1}^{p} \int_{t-\tau_{i}}^{t} \mathrm{e}^{\alpha(\theta-t)} u^{\top}(\theta) B_{i}^{\top} \mathrm{e}^{A^{\top}\left(t-\theta-\tau_{i}\right)} \\
& \times R \mathrm{e}^{A\left(t-\theta-\tau_{i}\right)} B_{i} u(\theta) \mathrm{d} \theta,
\end{aligned}
$$

is a Lyapunov-Krasovskii functional for the closed-loop system (3) in the sense of Lemma 1.

Proof. Proof of Item 1. We first choose two positive definite matrices $U, S \in \mathbf{R}^{n \times n}$ such that

$$
P_{11}=U+S, \quad P_{12}=P_{22}=S .
$$

Then the inequalities in (22) become

$$
\left[\begin{array}{ccc}
\frac{1}{p \tau_{i}}(\mathscr{P}+Q) & U B_{0} F+Q & \frac{1}{p \tau_{i}} U \\
F^{\top} B_{0}^{\top} U+Q & \Gamma_{22} & 0 \\
\frac{1}{p \tau_{i}} U & 0 & \Gamma_{33}(i)
\end{array}\right]<0
$$

in which $i \in \mathbf{I}[1, p], Q=S H+H^{\top} S+\mathscr{R}$ and $\mathscr{P}=$ $U\left(A+B_{0} F\right)+\left(A+B_{0} F\right)^{\top} U$. By using Lemma 4, we know that the inequalities in (26) are equivalent to

$$
\left[\begin{array}{cc}
\frac{1}{p \tau_{i}}(\mathscr{P}+Q)+\frac{1}{\tau_{i}} U\left(\mathrm{e}^{-\alpha \tau} R\right)^{-1} U & U B_{0} F+Q \\
F^{\top} B_{0}^{\top} U+Q & \Gamma_{22}
\end{array}\right]<0,
$$

for all $i \in \mathbf{I}[1, p]$. Clearly, there exists a matrix $R>0$ such that $\Gamma_{22}<0$ holds true under condition (19). By applying again Lemma 4, the inequalities in (27) are further equivalent to $\Gamma_{22}<0$, and

$$
\begin{aligned}
& \frac{1}{p \tau_{i}}(\mathscr{P}+Q)+\frac{1}{\tau_{i}} U\left(\mathrm{e}^{-\alpha \tau} R\right)^{-1} U \\
& -\left(U B_{0} F+Q\right) \Gamma_{22}^{-1}\left(U B_{0} F+Q\right)^{\top}<0,
\end{aligned}
$$

for all $i \in \mathbf{I}[1, p]$. From Assumption 1 we know that, for any $\varepsilon>0$ and $R>0$, the equation

$$
Q=S H+H^{\top} S+\mathscr{R}=-\varepsilon I_{n},
$$


has a solution $S>0$. Let $U=\varepsilon^{2} I_{n}$. Then we have, for all $i \in \mathbf{I}[1, p]$,

$$
\begin{aligned}
& \frac{1}{p \tau_{i}}(\mathscr{P}+Q)+\frac{1}{\tau_{i}} U\left(\mathrm{e}^{-\alpha \tau} R\right)^{-1} U \\
& -\left(U B_{0} F+Q\right) \Gamma_{22}^{-1}\left(U B_{0} F+Q\right)^{\top} \\
= & -\frac{\varepsilon}{p \tau_{i}} I_{n}+\varepsilon^{2}\left(\frac{1}{p \tau_{i}}\left(\left(A+B_{0} F\right)+\left(A+B_{0} F\right)^{\top}\right)-\Gamma_{22}^{-1}\right) \\
& +\varepsilon^{3}\left(B_{0} F \Gamma_{22}^{-1}+\Gamma_{22}^{-1} F^{\top} B_{0}^{\boldsymbol{\top}}\right) \\
& +\varepsilon^{4}\left(\frac{1}{\tau_{i}}\left(\mathrm{e}^{-\alpha \tau} R\right)^{-1}-B_{0} F \Gamma_{22}^{-1}(i) F^{\boldsymbol{\top}} B_{0}^{\boldsymbol{\top}}\right)
\end{aligned}
$$

Obviously, for all $i \in \mathbf{I}[1, p]$, if we let $\varepsilon$ be sufficiently small, the last three terms in (30) is dominated by the first term. Hence there exists an $\varepsilon^{*}>0$ such that the LMI's in (28) hold true for all $\varepsilon \in\left(0, \varepsilon^{*}\right]$. Finally, for the $P_{12}$ and $P_{22}$ given in (25), the inequality in (20) can be rewritten as

$$
S H+H^{\top} S+\mathscr{R}<0,
$$

which is obviously satisfied in view of (29). The proof of Item 1 is complete.

Proof of Item 2. It is obvious that there exists a scalar $c_{1}>0$ such that

$$
V\left(x(t), u_{t}\right) \geq c_{1}\left(|x(t)|^{2}+\left\|u_{t}\right\|^{2}\right) .
$$

It follows from Lemma 2 and Lemma 3 that inequality (33) holds true. So we have (34) for some $c_{2}>0$. To complete the proof, we only need to show

$$
\dot{V}\left(x(t), u_{t}\right) \leq-c_{3}\left(|x(t)|^{2}+\left\|u_{t}\right\|^{2}\right),
$$

for some $c_{3}>0$. From (10) we know that

$$
\begin{aligned}
\dot{\omega}(t) & =\sum_{i=1}^{p}\left(\mathrm{e}^{-A \tau_{i}} B_{i} u(t)-B_{i} u\left(t-\tau_{i}\right)\right. \\
& \left.+A \int_{t-\tau_{i}}^{t} \mathrm{e}^{A\left(t-\theta-\tau_{i}\right)} B_{i} u(\theta) \mathrm{d} \theta\right) .
\end{aligned}
$$

With this we can compute to get (37). Based on Item 1 of this theorem, we know that

$$
-\alpha R+A^{\top} R+R A<0
$$

is true. In addition, by using the Jensen inequality we have

$$
\begin{aligned}
& -\sum_{i=1}^{p} \mathrm{e}^{-\alpha \tau_{i}} u^{\top}\left(t-\tau_{i}\right) B_{i}^{\top} R B_{i} u\left(t-\tau_{i}\right) \\
& \leq-\sum_{i=1}^{p} u^{\top}\left(t-\tau_{i}\right) B_{i}^{\top}\left(\mathrm{e}^{-\alpha \tau} R\right) B_{i} u\left(t-\tau_{i}\right) \\
& \leq-\frac{1}{p}\left(\sum_{i=1}^{p} u^{\top}\left(t-\tau_{i}\right) B_{i}^{\top}\right) \mathrm{e}^{-\alpha \tau} R\left(\sum_{i=1}^{p} B_{i} u\left(t-\tau_{i}\right)\right) .
\end{aligned}
$$

Therefore, by applying (20) and (38), we can further obtain (39). Denote $\eta=\eta(t, \theta)$ where

$\eta=\left[x^{\top}(t), u^{\top}(\theta) B_{i}^{\top} \mathrm{e}^{A^{\top}\left(t-\theta-\tau_{i}\right)}, \sum_{i=1}^{p} u^{\top}\left(t-\tau_{i}\right) B_{i}^{\top}\right]^{\top}$.
Then (39) can be rewritten as

$$
\dot{V}\left(x(t), u_{t}\right) \leq \sum_{i=1}^{p} \int_{t-\tau_{i}}^{t} \eta^{\top}(t, \theta) \Gamma(i) \eta(t, \theta) \mathrm{d} \theta .
$$

By using the inequalities in (22) we know that there exists a sufficiently small number $\delta>0$ such that $\Gamma(i) \leq-\delta I_{3 n}$ for all $i \in \mathbf{I}[1, p]$. So we can further get (41) where $c_{3}$ is some constant. The proof is finished.

Remark 1 In [14], the definition of ISS LyapunovKrasovskii functional for coupled retarded differential equations and functional differential equations was given. Here we show that $V\left(x(t), u_{t}\right)$ is also an ISS LyapunovKrasovskii functional satisfying all the conditions in [14] if we define

$$
\begin{aligned}
\left\|u_{t}\right\| & =\sup _{\theta \in[-\tau, 0]}\{|u(t+\theta)|\}, \\
\left\|x_{t}\right\| & =\sup _{\theta \in[-\tau, 0]}\{|x(t+\theta)|\}, \forall t \geq 0 .
\end{aligned}
$$

In this case, it is clear from (34) that

$$
V\left(x(t), u_{t}\right) \leq \lambda_{\max }(P)\left\|x_{t}\right\|^{2}+\tau d_{1}\left\|u_{t}\right\|^{2},
$$

where

$$
\begin{aligned}
d_{1}= & \left(\lambda_{\max }(P) p^{2} \tau+p \lambda_{\max }(R)\right) \\
& \times \max _{i \in \mathbf{I}[1, p]}\left\{\left|B_{i}\right|^{2}\right\} \sup _{s \in[-\tau, 0]}\left\{\left|\mathrm{e}^{A s}\right|^{2}\right\} .
\end{aligned}
$$

In addition, it follows from (13) that $|u(t)| \leq|F||z(t)|$ and we can compute

$$
\begin{aligned}
& V\left(x(t), u_{t}\right)=\left[\begin{array}{l}
x(t) \\
z(t)
\end{array}\right]^{\top} P_{0}\left[\begin{array}{l}
x(t) \\
z(t)
\end{array}\right] \\
& +\sum_{i=1}^{p} \int_{t-\tau_{i}}^{t} \mathrm{e}^{\alpha(\theta-t)} u^{\top}(\theta) B_{i}^{\top} \mathrm{e}^{A^{\top}\left(t-\theta-\tau_{i}\right)} \\
& \times R \mathrm{e}^{A\left(t-\theta-\tau_{i}\right)} B_{i} u(\theta) \mathrm{d} \theta \\
& \geq \lambda_{\min }\left(P_{0}\right)\left(|x(t)|^{2}+|z(t)|^{2}\right) \\
& \geq \lambda_{\min }\left(P_{0}\right)|x(t)|^{2}+\frac{\lambda_{\min }\left(P_{0}\right)}{|F|^{2}}|u(t)|^{2},
\end{aligned}
$$

in which

$$
P_{0}=\left[\begin{array}{cc}
I_{n} & 0 \\
-I_{n} & I_{n}
\end{array}\right]^{\top} P\left[\begin{array}{cc}
I_{n} & 0 \\
-I_{n} & I_{n}
\end{array}\right]>0 .
$$

Moreover, it follows from (41) and (34) that

$$
\dot{V}\left(x(t), u_{t}\right) \leq-\frac{c_{3}}{c_{2}} V\left(x(t), u_{t}\right) .
$$

From the inequalities (44), (45) and (47) we know that $V\left(x(t), u_{t}\right)$ is also an ISS Lyapunov-Krasovskii functional [14].

\section{A Numerical Example}

In this section, a numerical example is given to validate the effectiveness of the proposed approach. We consider system (1) with parameters

$$
\begin{aligned}
A & =\left[\begin{array}{cc}
0.3316 & 0.7939 \\
-0.0576 & 1.0684
\end{array}\right], B_{0}=\left[\begin{array}{cc}
0.5000 & -0.9000 \\
1.0000 & 1.4000
\end{array}\right] \\
B_{1} & =\left[\begin{array}{cc}
-1.2000 & 2.0000 \\
0.7000 & 0.9000
\end{array}\right], B_{2}=\left[\begin{array}{cc}
-1.2000 & 1.8000 \\
2.5000 & 0.4000
\end{array}\right]
\end{aligned}
$$




$$
\begin{aligned}
\omega^{\boldsymbol{\top}}(t) \omega(t) & =\left(\sum_{i=1}^{p} \int_{t-\tau_{i}}^{t} \mathrm{e}^{A\left(t-\theta-\tau_{i}\right)} B_{i} u(\theta) \mathrm{d} \theta\right)^{\top}\left(\sum_{i=1}^{p} \int_{t-\tau_{i}}^{t} \mathrm{e}^{A\left(t-\theta-\tau_{i}\right)} B_{i} u(\theta) \mathrm{d} \theta\right) \\
& \leq p \sum_{i=1}^{p}\left(\int_{t-\tau_{i}}^{t} \mathrm{e}^{A\left(t-\theta-\tau_{i}\right)} B_{i} u(\theta) \mathrm{d} \theta\right)^{\top}\left(\int_{t-\tau_{i}}^{t} \mathrm{e}^{A\left(t-\theta-\tau_{i}\right)} B_{i} u(\theta) \mathrm{d} \theta\right) \\
& \leq p \tau \sum_{i=1}^{p} \int_{t-\tau_{i}}^{t} u^{\top}(\theta) B_{i}^{\top} \mathrm{e}^{A^{\top}\left(t-\theta-\tau_{i}\right)} \mathrm{e}^{A\left(t-\theta-\tau_{i}\right)} B_{i} u(\theta) \mathrm{d} \theta \\
& \leq p^{2} \tau \max _{i \in \mathbf{I}[1, p]}\left\{\left|B_{i}\right|^{2}\right\} \sup _{s \in[-\tau, 0]}\left\{\left|\mathrm{e}^{A s}\right|^{2}\right\} \int_{t-\tau}^{t}|u(\theta)|^{2} \mathrm{~d} \theta .
\end{aligned}
$$

$$
\begin{aligned}
V\left(x(t), u_{t}\right) & \leq \lambda_{\max }(P)\left(|x(t)|^{2}+|\omega(t)|^{2}\right)+p \lambda_{\max }(R) \max _{i \in \mathbf{I}[1, p]}\left\{\left|B_{i}\right|^{2}\right\} \sup _{s \in[-\tau, 0]}\left\{\left|\mathrm{e}^{A s}\right|^{2}\right\} \int_{t-\tau}^{t}|u(\theta)|^{2} \mathrm{~d} \theta \\
& \leq \lambda_{\max }(P)|x(t)|^{2}+\left(\lambda_{\max }(P) p^{2} \tau+p \lambda_{\max }(R)\right) \max _{i \in \mathbf{I}[1, p]}\left\{\left|B_{i}\right|^{2}\right\} \sup _{s \in[-\tau, 0]}\left\{\left|\mathrm{e}^{A s}\right|^{2}\right\} \int_{t-\tau}^{t}|u(\theta)|^{2} \mathrm{~d} \theta \\
& \leq c_{2}\left(|x(t)|^{2}+\left\|u_{t}\right\|^{2}\right)
\end{aligned}
$$

$$
\begin{aligned}
& \dot{V}\left(x(t), u_{t}\right)=2 x^{\top}(t) P_{11} \dot{x}(t)+2 \dot{\omega}^{\top}(t) P_{12}^{\top} x(t)+2 \omega^{\top}(t) P_{12}^{\top} \dot{x}(t)+2 \omega^{\top}(t) P_{22} \dot{\omega}(t) \\
& +\sum_{i=1}^{p} u^{\top}(t) B_{i}^{\top} \mathrm{e}^{-A^{\top} \tau_{i}} R \mathrm{e}^{-A \tau_{i}} B_{i} u(t)-\sum_{i=1}^{p} \mathrm{e}^{-\alpha_{i} \tau_{i}} u^{\top}\left(t-\tau_{i}\right) B_{i}^{\top} R B_{i} u\left(t-\tau_{i}\right) \\
& +\sum_{i=1}^{p} \int_{t-\tau_{i}}^{t} u^{\boldsymbol{\top}}(\theta) B_{i}^{\top} \mathrm{e}^{A^{\top}\left(t-\theta-\tau_{i}\right)}\left(\mathrm{e}^{\alpha(\theta-t)}\left(-\alpha R+A^{\boldsymbol{\top}} R+R A\right)\right) \mathrm{e}^{A\left(t-\theta-\tau_{i}\right)} B_{i} u(\theta) \mathrm{d} \theta, \\
& =x^{\boldsymbol{\top}}(t)\left(P_{11}\left(A+B_{0} F\right)+\left(A+B_{0} F\right)^{\boldsymbol{\top}} P_{11}+F^{\boldsymbol{\top}} \mathscr{B}^{\boldsymbol{\top}} P_{12}^{\boldsymbol{\top}}+P_{12} \mathscr{B} F+\mathscr{R}\right) x(t) \\
& +2 x^{\boldsymbol{\top}}(t)\left(A^{\boldsymbol{\top}} P_{12}+P_{12} A+P_{11} B_{0} F+P_{12} \mathscr{B} F+F^{\boldsymbol{\top}} B_{0}^{\boldsymbol{\top}} P_{12}+F^{\boldsymbol{\top}} \mathscr{B}^{\boldsymbol{\top}} P_{22}+\mathscr{R}\right) \omega(t) \\
& +2 x^{\boldsymbol{\top}}(t)\left(P_{11}-P_{12}\right) \sum_{i=1}^{p} B_{i} u\left(t-\tau_{i}\right)+2 \omega^{\boldsymbol{\top}}(t)\left(P_{12}^{\boldsymbol{\top}}-P_{22}\right) \sum_{i=1}^{p} B_{i} u\left(t-\tau_{i}\right) \\
& +\omega^{\top}(t) \Delta \omega(t)-\sum_{i=1}^{p} \mathrm{e}^{-\alpha \tau_{i}} u^{\top}\left(t-\tau_{i}\right) B_{i}^{\top} R B_{i} u\left(t-\tau_{i}\right) \\
& +\sum_{i=1}^{p} \int_{t-\tau_{i}}^{t} u^{\boldsymbol{\top}}(\theta) B_{i}^{\top} \mathrm{e}^{A^{\top}\left(t-\theta-\tau_{i}\right)} \mathrm{e}^{\alpha(\theta-t)}\left(-\alpha R+A^{\boldsymbol{\top}} R+R A\right) \mathrm{e}^{A\left(t-\theta-\tau_{i}\right)} B_{i} u(\theta) \mathrm{d} \theta .
\end{aligned}
$$

$$
\begin{aligned}
\dot{V}\left(x(t), u_{t}\right) & \leq x^{\boldsymbol{\top}}(t)\left(P_{11}\left(A+B_{0} F\right)+\left(A+B_{0} F\right)^{\boldsymbol{\top}} P_{11}+F^{\boldsymbol{\top}} \mathscr{B}^{\boldsymbol{\top}} P_{12}^{\boldsymbol{\top}}+P_{12} \mathscr{B} F+\mathscr{R}\right) x(t) \\
& +2 x^{\boldsymbol{\top}}(t)\left(A^{\boldsymbol{\top}} P_{12}+P_{12} A+P_{11} B_{0} F+P_{12} \mathscr{B} F+F^{\boldsymbol{\top}} B_{0}^{\boldsymbol{\top}} P_{12}+F^{\boldsymbol{\top}} \mathscr{B}^{\boldsymbol{\top}} P_{22}+\mathscr{R}\right) \omega(t) \\
& +2 x^{\boldsymbol{\top}}(t)\left(P_{11}-P_{12}\right) \sum_{i=1}^{p} B_{i} u\left(t-\tau_{i}\right)+2 \omega^{\boldsymbol{\top}}(t)\left(P_{12}^{\boldsymbol{\top}}-P_{22}\right) \sum_{i=1}^{p} B_{i} u\left(t-\tau_{i}\right) \\
& -\frac{1}{p}\left(\sum_{i=1}^{p} u^{\boldsymbol{\top}}\left(t-\tau_{i}\right) B_{i}^{\boldsymbol{\top}}\right) \mathrm{e}^{-\alpha \tau} R\left(\sum_{i=1}^{p} B_{i} u\left(t-\tau_{i}\right)\right) \\
& +\sum_{i=1}^{p} \int_{t-\tau_{i}}^{t} u^{\boldsymbol{\top}}(\theta) B_{i}^{\boldsymbol{\top}} \mathrm{e}^{A^{\boldsymbol{\top}}\left(t-\theta-\tau_{i}\right)} \mathrm{e}^{-\alpha \tau}\left(-\alpha R+A^{\boldsymbol{\top}} R+R A\right) \mathrm{e}^{A\left(t-\theta-\tau_{i}\right)} B_{i} u(\theta) \mathrm{d} \theta .
\end{aligned}
$$

and

$$
B_{3}=\left[\begin{array}{cc}
0.7000 & -0.2000 \\
0.9000 & 1.4000
\end{array}\right],
$$

and $\tau_{1}=0.2, \tau_{2}=0.4, \tau_{3}=1$. If we choose

$$
F=\left[\begin{array}{cc}
-0.2936 & -0.0442 \\
0.2764 & -0.5956
\end{array}\right],
$$




$$
\begin{aligned}
\dot{V}\left(x(t), u_{t}\right) & \leq-\delta \sum_{i=1}^{p} \int_{t-\tau_{i}}^{t} \eta^{\top}(t, \theta) \eta(t, \theta) \mathrm{d} \theta \\
& \leq-\delta \sum_{i=1}^{p} \int_{t-\tau_{i}}^{t}\left(|x(t)|^{2}+\left|\mathrm{e}^{A\left(t-\theta-\tau_{i}\right)} B_{i} u(\theta)\right|^{2}\right) \mathrm{d} \theta \\
& \leq-\delta \int_{t-\tau}^{t}\left(|x(t)|^{2}+\left|\mathrm{e}^{A(t-\theta-\tau)} B_{p} u(\theta)\right|^{2}\right) \mathrm{d} \theta \\
& \leq-\delta\left(\tau|x(t)|^{2}+\inf _{s \in[-\tau, 0]}\left\{\lambda_{\min }\left(B_{p}^{\top} \mathrm{e}^{A^{\top} s} \mathrm{e}^{A s} B_{p}\right)\right\} \int_{t-\tau}^{t}|u(\theta)|^{2} \mathrm{~d} \theta\right) \\
& \leq-c_{3}\left(|x(t)|^{2}+\left\|u_{t}\right\|^{2}\right),
\end{aligned}
$$

then Assumption 1 holds true since $\lambda(H)=\{-0.2,-0.5\}$. Notice that $\lambda(A)=\{1,0.4\}$. By taking $\alpha=2.1$ and solving the set of LMIs in Theorem 1 we can get

$$
\begin{aligned}
& P=\left[\begin{array}{cccc}
30.2616 & -35.2265 & 30.5653 & -35.5330 \\
-35.2265 & 51.5767 & -35.5462 & 51.9109 \\
30.5653 & -35.5330 & 31.2272 & -36.2693 \\
-35.5462 & 51.9109 & -36.2693 & 52.7382
\end{array}\right], \\
& R=\left[\begin{array}{cc}
40.7077 & -44.8847 \\
-44.8847 & 59.8366
\end{array}\right] .
\end{aligned}
$$

Therefore, a Lyapunov-Krasovskii functional in the form of (23) with $P$ and $R$ given above for system (1) with parameters (48) can be constructed accordingly.

\section{Conclusion}

This paper considered the Lyapunov-Krasovskii functionals for predictor feedback systems with multiple input delays. For linear systems with multiple input delays controlled by the predictor feedback, based on the equivalent delay-free systems, a Lyapunov-Krasovskii functional was constructed by solving a set of LMIs, which were shown to be solvable as long as the closed-loop system is asymptotically stable. In addition, it is shown that the obtained Lyapunov-Krasovskii functionals is also an ISS LyapunovKrasovskii functionals for the closed-loop systems.

\section{References}

[1] Z. Artstein, Linear systems with delayed controls: a reduction, IEEE Transactions on Automatic Control, Vol. 27, No. 4, pp. 869-879, 1982.

[2] N. Bekiaris-Liberis and M. Krstic, Lyapunov stability of linear predictor feedback for distributed input delays, IEEE Transactions on Automatic Control, Vol. 56, No. 3, pp. 655660, 2011.

[3] H. H. Choi, M. J. Chung, Memoryless stabilization of uncertain dynamic systems with time-varying delayed states and controls, Automatica, Vol. 31, No. 9, pp. 1349-1351, 1995.

[4] K. Gu, Discretized LMI set in the stability problem of linear uncertain time-delay systems, International Journal of Control, Vol. 68, No. 4, pp. 923-934, 1997.

[5] J. H. Kim, E. T. Jeung and H. B. Park, Robust control for parameter uncertain delay systems in state and control input, Automatica, Vol. 32, No. 9, pp. 1337-1339, 1996.

[6] M. Krstic, Lyapunov stability of linear predictor feedback for time-varying input delay, IEEE Transactions on Automatic Control, Vol. 55, No. 2, pp. 554-559, 2010.
[7] O. M. Kwona, J. H. Park, S. M. Lee, On stability criteria for uncertain delay-differential systems of neutral type with timevarying delays, Applied Mathematics and Computation, Vol. 197, No. 2, pp. 864-873, 2008.

[8] X. Li, Carlos E. De Souza, Criteria for robust stability and stabilization of uncertain linear systems with state delay, $\mathrm{Au}$ tomatica, Vol. 33, No. 9, 1657-1662, 1997.

[9] Z.-Y. Li, Z. Lin and B. Zhou, An analysis of the exponential stability of linear stochastic neutral delay systems, International Journal of Robust and Nonlinear Control, DOI: 10.1002/rnc.3058.

[10] Z.-Y. Li, B. Zhou and Z. Lin, On robustness of predictor feedback control of linear systems with input delays, Automatica, Vol. 50, No. 5, pp. 1497-1506, 2014.

[11] D. Liu, X. Liu, S. Zhong, Delay-dependent robust stability and control synthesis for uncertain switched neutral systems with mixed delays, Applied Mathematics and Computation, Vol. 202, No. 2, pp. 828-839, 2008.

[12] R. Lozano, P. Castillo, P. Garcia, A. Dzul, Robust predictionbased control for unstable delay systems: application to the yaw control of a mini-helicopter, Automatica, Vol. 40, No. 4, pp. 603-612, 2004.

[13] D. Q. Mayne, Control of linear systems with time delay, Electronics Letters, Vol. 4, No. 20,

[14] F. Mazenc, N. Silviu-Iulian and M. Krstic, LyapunovKrasovskii functionals and application to input delay compensation for linear time-invariant systems, Automatica, Vol. 48, No. 7 pp. 1317-1323, 2012.

[15] P. Pepe, Z-P Jiang, A Lyapunov-Krasovskii methodology for ISS and iISS of time-delay systems, Systems \& Control Letters, Vol. 55, No. 12, pp. 1006-1014, 2006.

[16] R. Samli, S. Arik, New results for global stability of a class of neutral-type neural systems with time delays, Applied Mathematics and Computation, Vol. 210, No. 2, pp. 564-570, 2009.

[17] S. Xu, J. Lam, C. Yang, Quadratic stability and stabilization of uncertain linear discrete-time systems with state delay, Systems \& Control Letters, Vol. 43, No. 2, pp. 77-84, 2001.

[18] D. Yue, Q-L Han, Delayed feedback control of uncertain systems with time-varying input delay, Automatica, Vol. 41, No. 2, pp. 233-240, 2005.

[19] B. Zhou and Z. Lin, Truncated predictor feedback stabilization of polynomially unstable linear systems with multiple time-varying input delays, IEEE Transactions on Automatic Control, DOI: 10.1109/TAC.2013.2297188. 